\title{
Effects of chito-oligosaccharides supplementation on performance, nutrient digestibility, pork quality and immune response in growing-finishing pigs*
}

\author{
K.N. Han ${ }^{1}$, Y.X. Yang ${ }^{1}$, T.W. Hahn' ${ }^{2}$, I.K. Kwon ${ }^{3}$, J. D. Lohakare', \\ J.K. Lee ${ }^{1}$ and B.J. Chae ${ }^{1,4}$
}

\author{
Kangwon National University, \\ ${ }^{1}$ Department of Animal Resources Science, \\ ${ }^{2}$ Department of Veterinary Medicine, \\ ${ }^{3}$ Department of Animal Product and Food Science \\ Chuncheon 200-701, Republic of Korea
}

(Received 6 February 2007; revised version 12 November 2007; accepted 21 November 2007)

\begin{abstract}
Two experiments were conducted to study the effects of chito-oligosaccharides (COS) on pig's performance and immune response. In Experiment 1, a total of 117 Landrace $\times$ Yorkshire $\times$ Duroc pigs (with average body weight of $30.7 \pm 1.56 \mathrm{~kg}$ ) were allocated to three treatments based on body weight to evaluate the effect of COS on growth performance, nutrient digestibility and pork quality. Treatments were control (no COS), T1 [0.04\% COS for grower $(30-55 \mathrm{~kg}), 0.02 \%$ for early finisher $(55-85 \mathrm{~kg})$ and $0.02 \%$ for late finisher $(85-110 \mathrm{~kg})]$, and T2 [0.1\% COS for grower $(30-55 \mathrm{~kg})$, $0.05 \%$ for early finisher $(55-85 \mathrm{~kg})$ and $0.025 \%$ for late finisher $(85-110 \mathrm{~kg})]$, respectively. Each treatment had 3 replications with 13 pigs per replicate. At the end of feeding trial, 2 pigs per replicate (6 per treatment) were slaughtered for carcass analysis and pork quality evaluation. During the overall period $(30-110 \mathrm{~kg})$, the growth performance and apparent nutrient digestibility were not affected $(\mathrm{P}>0.05)$ by dietary treatments. However, FCR was improved by $5.9 \%$ in $\mathrm{T} 2$ group when compared with control. Dressing percentage, backfat thickness, pork colour and marbling score were not influenced by the addition of COS. With the increase of storage time, TBARS was decreased and drip loss, pork colour were improved by the addition of COS. Linear reduction $(\mathrm{P}<0.10)$ in the LDL content of pork with the addition of COS was noted. In Experiment 2, fifty-four Landrace $\times$ Yorkshire $\times$ Duroc pigs (with average body weight of $30.9 \pm 1.78 \mathrm{~kg}$ ) were penned ( 6 pigs per pen, 3 pens per treatment) and allocated to three treatment diets for immune response study. The diets fed were: $0 \% \operatorname{COS}$ (control), $0.1 \% \operatorname{COS}$ (T1) and $0.3 \%$ COS (T2). During the $70-\mathrm{d}$ study, 3 pigs per replicate were vaccinated with Actinobacillus pleuropneumoniae and Pasteurella multocida and the antibody

\footnotetext{
* Supported in part by the Institute of Animal Resources at Kangwon National University and Kunpoong Bio Company, Seoul, Korea

${ }^{4}$ Corresponding author: e-mail: bjchae@kangwon.ac.kr
} 
titers were measured at $\mathrm{d} 0,5,30,50$ and 70 . Higher $(\mathrm{P}<0.05)$ antibody titers were observed for both of the COS added diets when compared with control. Flow cytometry was used to determine porcine lymphocyte subpopulations at $28^{\text {th }}$ and $54^{\text {th }}$ day from the other 3 unvaccinated pigs of each replicate. There was a quadratic increase $(\mathrm{P}<0.10)$ in MHC class-II and B-cells at $54^{\text {th }}$ day in pigs fed COS diets. In conclusion, feeding chito-oligosaccharides in growing-finishing pigs improved pork stability and reduced cholesterol in pork without affecting growth performance, and the immune response was improved by chito-oligosaccharides supplementation.

KEY WORDS: chito-oligosaccharides, performance, pork quality, immunity, growing-finishing pigs

\section{INTRODUCTION}

Certain types of oligosaccharides have been used as prebiotics to improve animal performance, enhance immunity and affect gut microflora. Chitosan is a copolymer consisting of $\beta$ - $(1 \rightarrow 4)-2$-acetamido-D-glucose and $\beta-(1 \rightarrow 4)-2$ amino-D-glucose units that are derived from chitin by deacetylation with alkali (Arvanitoyannis et al., 1998). Recent studies indicated that converting chitin and chitosan to water-soluble oligosaccharides improved its biological activities. Chito-oligosaccharide (COS) is an oligosaccharide that is easily obtained by chemical and enzymatic hydrolysis of poly-chitosan. Poly-chitosan is the second most abundant carbohydrate polymer found in nature. However, its insolubility and high viscosity limit its application for use with animals as a nutrient source. In contrast, COS has low molecular weight, good solubility, and low viscosity.

The health benefits of COS have recently been identified. The component was shown to have antimicrobial, anitifungal and antitumor activities and improved immune response in animals (Wu et al., 2006). It has also been shown to reduce blood cholesterol and triglyceride in early-weaned pigs or obese diabetic mice (Tang et al., 2005). However, its role in regulating the blood lipid content is still controversial (Ikeda et al., 1993).

These days, in the production of pork meat, the general aim is to provide a product that is safe and of good quality to meet the customer's needs. Many studies have been done on developing agents to improve meat quality, with emphasis on improving the efficiency of producing lean carcass and preventing lipid oxidation. Kim et al. (1999) found carcass dressing percentage and backfat thickness were improved when supplemented with chitosan compared to antibiotics in pigs. Furthermore, studies reported that chitin and chitosan improved pork water-holding capacity and reduced blood cholesterol (Ikeda et al., 1993). However, the effect of COS on meat quality has not yet been evaluated. Therefore, the objective of current studies was to investigate effects of supplemental COS on growth performance, nutrient digestibility, pork quality and immune response in growingfinishing pigs. 


\section{MATERIAL AND METHODS}

\section{Experiment 1}

\section{Experimental design, animals and procedures}

A total of 117 crossbred pigs (Landrace $\times$ Yorkshire $\times$ Duroc; average body weight of $30.7 \pm 1.56 \mathrm{~kg}$ ) were used to investigate the effect of chito-oligosaccharide on growth performance, nutrient digestibility and pork quality. Pigs were randomly allocated to three treatments based on body weight in a single factorial arrangement. Each treatment had 3 replications with 13 pigs per replicate. Treatments were control (no COS), T1 [0.04\% COS for grower $(30-55 \mathrm{~kg}), 0.02 \%$ COS for early finisher $(55-85 \mathrm{~kg})$ and $0.02 \%$ COS for late finisher $(85-110 \mathrm{~kg})]$, and T2 [0.1\% COS for grower $(30-55 \mathrm{~kg}), 0.05 \%$ COS for early finisher (55-85 $\mathrm{kg}$ ) and $0.025 \%$ COS for late finisher $(85-110 \mathrm{~kg})]$. The COS used in this study was obtained from chitin of the crab shell after deacetylation with concentrated sodium hydroxide at high temperature and then further decomposition by chitosanase-KPB enzyme in presence of ascorbic acid (Kunpoong Bio Co, Seoul). This product, named Biovita- $\mathrm{P}^{\circledR}$, was used in the present study.

The basal formula and chemical composition of experimental diets are presented in Table 1. COS replaced maize on equal percent basis for all the phases. All the diets met or exceed the nutrient requirements as recommended by NRC (1998). The pigs were housed in a $4.0 \mathrm{~m} \times 2.8 \mathrm{~m}$ pens with half slatted floors. Feed and water were offered ad libitum. The temperature range in the farm varied from 18 to $26^{\circ} \mathrm{C}$. During the study, body weight and feed intake were recorded at the end of each phase. Average daily gain (ADG), average daily feed intake (ADFI) and feed conversion ratio (FCR) were calculated at the end of each phase of the feeding trial.

Two digestibility trials were conducted using chromic oxide $(0.25 \%)$ as an indicator during grower and early finisher phases, respectively. All pigs in all pens were fed diets mixed with chromic oxide and faecal grab samples were collected from all pigs at respective phases of the study. The trails lasted for 10 days and faecal samples were collected from day 8 to 10 . The samples were pooled and dried in a forced-air drying oven at $60^{\circ} \mathrm{C}$ for $72 \mathrm{~h}$, then ground with a $1 \mathrm{~mm}$ mesh Wiley mill for chemical analysis.

At the end of feeding trial, 2 pigs per replicate reflecting average body weights of the same sex ( 6 males per treatment) were weighed just before immobilization, then exsanguinated, scalded, dehaired, decapitated, eviscerated, halved and inspected for carcass analysis and pork quality evaluation. All loins were cut into 2.54-cm-thick chops. Chops were deboned and trimmed of subcutaneous fat, and then chops were paired and placed in vacuum bags. The vacuum packages were assigned to 5 or 10 day of cold $\left(4^{\circ} \mathrm{C}\right)$ storage. From each loin, three 
Table 1. Formula and chemical composition of experiment diets for feeding trial

\begin{tabular}{|c|c|c|c|}
\hline \multirow[b]{2}{*}{ Items } & \multirow{2}{*}{$\begin{array}{c}\text { Grower } \\
30-55 \mathrm{~kg}\end{array}$} & \multicolumn{2}{|c|}{ Finisher } \\
\hline & & $\begin{array}{c}\text { early } \\
55-85 \mathrm{~kg}\end{array}$ & $\begin{array}{c}\text { late } \\
85-110 \mathrm{~kg}\end{array}$ \\
\hline \multicolumn{4}{|l|}{ Ingredients, $\%$} \\
\hline maize & 51.44 & 68.12 & 63.68 \\
\hline soyabean meal $(44 \%)$ & 29.80 & 21.08 & 16.40 \\
\hline rice bran & 7.00 & 2.42 & - \\
\hline wheat bran & - & - & 13.00 \\
\hline animal fat & 4.00 & 3.00 & 2.40 \\
\hline molasses & 3.00 & 3.00 & 2.00 \\
\hline rapeseed meal & 2.00 & - & - \\
\hline $\mathrm{TCP}$ & 1.50 & 0.30 & 0.82 \\
\hline limestone & 0.40 & 1.57 & - \\
\hline L-lysine $(78 \%)$ & 0.07 & 0.01 & 0.06 \\
\hline DL-methionine (100\%) & 0.02 & - & - \\
\hline vitamin $_{\text {premix }}{ }^{1}$ & 0.12 & 0.10 & 0.10 \\
\hline mineral premix ${ }^{2}$ & 0.20 & 0.10 & 0.20 \\
\hline salt & 0.30 & 0.30 & 0.30 \\
\hline choline $(25 \%)$ & 0.05 & - & 0.04 \\
\hline CTC & 0.10 & - & - \\
\hline \multicolumn{4}{|l|}{ Chemical composition $^{3}$} \\
\hline $\mathrm{ME}, \mathrm{kcal} / \mathrm{kg}$ & 3.300 & 3.300 & 3.200 \\
\hline crude protein, $\%$ & 19.05 & 15.00 & 14.47 \\
\hline lysine, $\%$ & 1.10 & 0.82 & 0.75 \\
\hline Met + Cys, $\%$ & 0.65 & 0.71 & 0.52 \\
\hline $\mathrm{Ca}, \%$ & 0.78 & 0.75 & 0.65 \\
\hline available $\mathrm{P}, \%$ & 0.39 & 0.38 & 0.25 \\
\hline \multicolumn{4}{|c|}{$\begin{array}{l}{ }^{1} \text { supplied per kg diet; } \mathrm{IU} \text { : vit. A } 12.000 \text {; vit. } \mathrm{D}_{3} 3.000 \text {, vit. E } 30 \text {; mg: vit. } \mathrm{K}_{3} 3.45 \text {, vit. } \mathrm{B}_{1} 1.8 \text {, } \\
\text { 14.4, vit. } \mathrm{B}_{6} 3 \text {, vit. } \mathrm{B}_{12} 0.045 \text {, pantothenic acid } 30 \text {, niacin } 90 \text {, biotin } 0.105 \text {, folic acid } 0.75 \\
{ }^{2} \text { supplied per kg diet; } \mathrm{mg} \text { : Cu } 143, \mathrm{Fe} 125, \mathrm{Zn} 102, \mathrm{Mn} 38.74 \text {, Co } 0.75 \text {, I } 0.75 \text {, Se } 0.23\end{array}$} \\
\hline
\end{tabular}

1.27-cm chops were also cut for the measurements of lipid oxidation (TBARS) and cholesterol. These chops were stored in vacuum bags under the same conditions as the $2.54-\mathrm{cm}$ chops.

\section{Chemical analysis and carcass traits evaluation}

Analysis of the experimental diets and excreta was done according to the AOAC (1990). Gross energy was measured by a bomb calorimeter (Model 1261, Parr Instrument Co., Molin, IL, USA), and chromium with an automated spectrophotometer (Shimadzu, Japan). 
After slaughter, carcass traits were also evaluated. Dressing percentage was determined by the following equation: (hot carcass weight/ final liveweight) $\times$ 100. Liveweight was monitored the day before slaughter. Backfat thickness was determined at the $10^{\text {th }} \mathrm{rib}$, at three quarters of the lateral length of the loin muscle perpendicular. Chops were removed from the longissmuss thoracis at lumborum starting at the $11^{\text {th }}$ rib location and continued towards the caudal end for drip loss (one $2.5-\mathrm{cm}$ thick chop) determination. At $24 \mathrm{~h}$ post-mortem, whole loins were subjectively evaluated for colour, marbling between the $10^{\text {th }}$ and $11^{\text {th }}$ rib face according to a 5-point descriptive scale by the National Pork Producers Council Quality Standards. In addition, $\mathrm{L}^{*}, \mathrm{a}^{*}$, and $\mathrm{b}^{*}$ colour values were measured using colour difference meter (Yasuda Seiko Co., CR-310, Minolta, Japan) at 0, 5, or 10 day of storage at $4^{\circ} \mathrm{C}$. Samples of each chop were measured for lipid oxidation at 0,5 and 10 day of storage. Lipid oxidation of loin chops was measured by TBARS analysis as described previously by Sinnhuber and Yu (1977).

The lion chops samples were frozen in liquid $\mathrm{N}$ and stored at $-30^{\circ} \mathrm{C}$ until they were analysed for total cholesterol concentration, high-density lipoprotein (HDL) and low-density lipoprotein (LDL). Total muscle cholesterol content was determined with the enzymatic method. HDL was determined by using HDL cholesterol assay kit (Sigma-Aldrich, Seoul, Korea).

\section{Experiment 2}

\section{Animals, diets and procedures}

Fifty-four pigs (Landrace $\times$ Yorkshire $\times$ Duroc; with average body weight of $30.9 \pm 1.78 \mathrm{~kg}$ ) were allocated to three treatments for immune response studies. Each treatment had 3 replicates and each replicate had 6 pigs per pen. The diets fed were: $0 \% \operatorname{COS}$ (control), $0.1 \% \operatorname{COS}(\mathrm{T} 1)$ and $0.3 \% \operatorname{COS}(\mathrm{T} 2)$. The formula and chemical composition of experimental diets are presented in Table 1 and COS was added by replacing maize on equal percent basis. During the 70-d study, 3 pigs per replicate randomly selected were vaccinated with formalin inactivated vaccine of Actinobacillus pleuropneumoniae and Pasteurella multocida $(2 \mathrm{ml} \mathrm{I} /$ $\mathrm{M}$ ) and antibody titers were measured at $\mathrm{d}$ 0, 5, 30, 50 and 70 with ELISA (JeilBio Co., Korea). The pigs were housed in a $3.0 \times 2.8 \mathrm{~m}$ pens with concrete floors. Blood was drawn from the jugular vein at respective intervals post vaccination using a 22-gauge sterile needle into a $10 \mathrm{ml}$ syringe. The blood was centrifuged at $1500 \mathrm{~g}$ for $10 \mathrm{~min}$ and serum was collected and stored for further analysis.

ELISA was conducted by manufacturer's protocol (Jeil-Bio Co., Korea). Briefly, formalin inactivated $P$. multocida or A. pleuropneumoniae whole cells were coated in 96 well ELISA plate. Two-fold diluted sera were dispensed into each well and incubated for $2 \mathrm{~h}$. After washing three times with washing buffer, 
anti-pig IgG peroxidase conjugate (Sigma Chemical, St. Louis, USA) was added to each well. $o$-phenylenediamine was used as substrate and absorbance was measured at $490 \mathrm{~nm}$ with ELISA reader (Microplate autoreader, Bio-Tek Instruments, Winooski, VT).

\section{Lymphocyte subpopulation assay}

During the study, the effects of COS on porcine lymphocyte populations were also measured using fluorescence-activated cell sorter (FACS, Becton Dickinson, Franklin Lakes, NJ, USA). Monoclonal antibodies specifically reactive with porcine major histo-compatibility complex (MHC) class II, cluster of differentiation antigens 2, 4 and $8(\mathrm{CD} 2, \mathrm{CD} 4$, and CD8, respectively) B-cells, Non-T/Non-B cells (N), and granulocytes $(\mathrm{G})$ were measured. The blood was drawn from the other 3 pigs per pen (nonvaccinated) at $28^{\text {th }}$ and $54^{\text {th }}$ day of feeding and leukocyte population was measured using flow cytometry. Blood was drawn from the jugular vein by using 22 -gauge sterile needle into a $10 \mathrm{ml}$ syringe and then transferred to the tube containing same volume of acid citrate dextrose (ACD) solution ( $25 \mathrm{~m} M$ citric acid, $51.7 \mathrm{~m} M$ sodium acetate, $81.6 \mathrm{~m} M$ D-glucose) as an anticoagulant and stored at $4^{\circ} \mathrm{C}$ until analysis.

Six milliliters of blood mixed with ACD was centrifuged at $1500 \mathrm{~g}$ for 10 min and white blood cells (WBC) were collected with a sterile Pasteur pipette and placed on the surface of Hypaque Ficoll (Histopaque 1.803, Sigma Chemical Co., St. Louis, USA) and centrifuged at $500 \mathrm{~g}$ for $30 \mathrm{~min}$. The lymphocytes were obtained from the interface between Ficoll and plasma, and cell suspensions were washed three times and resuspended in a $\mathrm{Ca}$ and $\mathrm{Mg}$ free phosphate buffered saline (11.3 $\mathrm{m} M$ sodium phosphate, $3.8 \mathrm{~m} M$ potassium phosphate, $125 \mathrm{~m} M$ sodium chloride, 100 units $/ \mathrm{ml}$ penicillin and $100 \mu \mathrm{g} / \mathrm{ml}$ streptomycin).

Cells were then incubated with a panel of monoclonal antibodies (mAb), obtained from Seoul National University, Seoul, specific for various swine leukocyte differentiation antigen markers. The panel of mAb included PT85A (MHC class $\mathrm{II}$ ), $\mathrm{H} 42 \mathrm{~A}$ (IgG2a), and $\mathrm{mAb}$ specific for porcine CD2, CD4, CD8, and $\mathrm{B}$ and $\mathrm{N}$ cells. Lymphocytes were subtyped by the flow cytometry CellQuest program and cell populations were counted and analysed with the FACS Calibur and CellQuest program (Koo et al., 2006).

\section{Statistical analysis}

Statistical analysis was conducted by using the GLM procedure of SAS software (1996). The treatments were the main effects. Pen was used as an experimental unit for all analysis. Linear and quadratic polynomials were evaluated for increasing COS levels. The level of significance was accepted at $0.10 \%$ level, unless otherwise noted. 


\section{RESULTS}

There were no effects (linear or quadratic; $\mathrm{P}>0.05$ ) of COS supplementation on the ADG, ADFI and FCR during grower, early-finisher, late-finisher and the overall period (Table 2).

Table 2. Effect of chito-oligosaccharides on the growth performance of growing-finishing pigs

\begin{tabular}{|c|c|c|c|c|c|c|}
\hline \multirow{2}{*}{ Items } & \multicolumn{3}{|c|}{ Treatments $^{1}$} & \multirow{2}{*}{$\mathrm{SEM}^{2}$} & \multicolumn{2}{|c|}{ P-value } \\
\hline & control & $\mathrm{T} 1$ & $\mathrm{~T} 2$ & & linear & quadratic \\
\hline \multicolumn{7}{|c|}{ Grower, $30-55 \mathrm{~kg}$} \\
\hline ADG, $\mathrm{g}$ & 704 & 722 & 718 & 9.27 & 0.5909 & 0.6085 \\
\hline ADFI, $g$ & 1.703 & 1.749 & 1.647 & 59.68 & 0.7444 & 0.6227 \\
\hline FCR & 2.42 & 2.42 & 2.30 & 0.07 & 0.5489 & 0.7406 \\
\hline \multicolumn{7}{|c|}{ Early finisher, $55-85 \mathrm{~kg}$} \\
\hline ADG, $\mathrm{g}$ & 735 & 741 & 738 & 8.21 & 0.8875 & 0.8338 \\
\hline ADFI, $g$ & 2.365 & 2.407 & 2.367 & 35.39 & 0.9864 & 0.6516 \\
\hline FCR & 3.22 & 3.26 & 3.20 & 0.05 & 0.9120 & 0.7316 \\
\hline \multicolumn{7}{|c|}{ Late finisher, $85-110 \mathrm{~kg}$} \\
\hline ADG, $\mathrm{g}$ & 858 & 886 & 879 & 6.79 & 0.2271 & 0.2443 \\
\hline ADFI, $g$ & 3.140 & 3.124 & 3.146 & 46.11 & 0.9644 & 0.8703 \\
\hline FCR & 3.66 & 3.53 & 3.58 & 0.05 & 0.5412 & 0.4057 \\
\hline \multicolumn{7}{|c|}{ Overall, $30-110 \mathrm{~kg}$} \\
\hline ADG, $\mathrm{g}$ & 724 & 759 & 738 & 8.58 & 0.4807 & 0.1553 \\
\hline ADFI, $g$ & 2.089 & 2.170 & 2.013 & 78.08 & 0.7291 & 0.5404 \\
\hline FCR & 2.90 & 2.87 & 2.73 & 0.12 & 0.6363 & 0.8502 \\
\hline
\end{tabular}

${ }^{1}$ control: no chito-oligosaccharides; T1: chito-oligosaccharides added at $0.04 \%$ (30-55 kg), $0.02 \%$ $(55-85 \mathrm{~kg}), 0.02 \%(85-110 \mathrm{~kg})$, respectively; T2: chito-oligosaccharides added at $0.1 \%(30-55 \mathrm{~kg})$, $0.05 \%(55-85 \mathrm{~kg}), 0.025 \%(85-110 \mathrm{~kg})$, respectively

${ }^{2}$ standard error of means

The apparent nutrient digestibility was also not influenced $(\mathrm{P}>0.05)$ by COS addition in diets during grower and early finisher phase (Table 3 ). However, the digestibility of GE was quadratically $(\mathrm{P}=0.0810)$ and linearly $(\mathrm{P}=0.0704)$ decreased with increase in the inclusion of COS during grower and early finisher periods, respectively.

The carcass traits like dressing percentage, backfat thickness, lean percent, pork colour score and marbling score did not reveal any linear or quadratic effect $(\mathrm{P}>0.05)$ by dietary COS supplementation (Table 4$)$. There was also no difference in TBARS value of meat at day 0 and 5 due to addition of COS, however, both linear $(\mathrm{P}=0.0048)$ and quadratic $(\mathrm{P}=0.0020)$ effects were noticed at day 10 showing reduction in TBARS values in COS added diets. 
Table 3. Effect of chito-oligosaccharides on apparent fecal nutrient digestibility of growing-finishing pigs

\begin{tabular}{|c|c|c|c|c|c|c|}
\hline \multirow{2}{*}{ Items } & \multicolumn{3}{|c|}{ Treatments $^{1}$} & \multirow{2}{*}{ SEM $^{2}$} & \multicolumn{2}{|c|}{ P-value } \\
\hline & control & $\mathrm{T} 1$ & $\mathrm{~T} 2$ & & linear & quadratic \\
\hline \multicolumn{7}{|c|}{ Grower } \\
\hline $\mathrm{DM}$ & 79.88 & 78.01 & 79.85 & 0.69 & 0.9984 & 0.2680 \\
\hline GE & 80.11 & 77.73 & 79.89 & 0.58 & 0.8620 & 0.0810 \\
\hline $\mathrm{CP}$ & 75.95 & 71.84 & 72.85 & 1.08 & 0.2609 & 0.2812 \\
\hline $\mathrm{EE}$ & 69.44 & 78.97 & 79.17 & 3.21 & 0.2629 & 0.5193 \\
\hline $\mathrm{Ca}$ & 55.57 & 57.13 & 52.34 & 1.01 & 0.1627 & 0.1211 \\
\hline $\mathrm{P}$ & 55.83 & 52.23 & 56.30 & 1.47 & 0.9031 & 0.2794 \\
\hline \multicolumn{7}{|c|}{ Early finisher } \\
\hline $\mathrm{DM}$ & 80.78 & 78.91 & 79.28 & 0.41 & 0.1250 & 0.1753 \\
\hline GE & 78.74 & 76.75 & 76.50 & 0.51 & 0.0704 & 0.3642 \\
\hline $\mathrm{CP}$ & 77.01 & 75.40 & 76.64 & 0.58 & 0.8154 & 0.3166 \\
\hline $\mathrm{EE}$ & 49.09 & 47.90 & 48.28 & 0.74 & 0.7050 & 0.6710 \\
\hline $\mathrm{Ca}$ & 46.87 & 57.99 & 57.25 & 2.64 & 0.1073 & 0.2583 \\
\hline $\mathrm{P}$ & 37.05 & 41.76 & 40.25 & 1.85 & 0.5341 & 0.4870 \\
\hline
\end{tabular}

${ }^{1}$ control: no chito-oligosaccharides; T1: chito-oligosaccharides added at $0.04 \%(30-55 \mathrm{~kg}), 0.02 \%$ $(55-85 \mathrm{~kg}), 0.02 \%(85-110 \mathrm{~kg})$, respectively; T2: chito-oligosaccharides added at $0.1 \%(30-55 \mathrm{~kg})$, $0.05 \%(55-85 \mathrm{~kg}), 0.025 \%(85-110 \mathrm{~kg})$, respectively

${ }^{2}$ standard error of means

The drip loss was not affected $(\mathrm{P}>0.05)$ by dietary COS supplementation at day 5 ; however, there was a linear reduction $(\mathrm{P}=0.0530)$ in drip loss as the increasing of dietary COS content at day 10 . The CIE values at day 0 and 5 remained unaffected $(\mathrm{P}>0.05)$, while at day 10 of refrigerated storage, there was a linear increase in $\mathrm{a}^{*}$ values $(\mathrm{P}=0.0546)$ and decrease in $\mathrm{b}^{*}$ values $(\mathrm{P}=0.0001)$ with increase in the inclusion of COS, respectively (Table 4).

The pork LDL cholesterol was reduced (linear; $\mathrm{P}=0.0634$ ) with the increase in COS level in diets (Table 5), however, the total and HDL cholesterol in pork remained unaffected.

The antibody titers to A. pleuropneumoniae measured by ELISA showed linear increment $(\mathrm{P}<0.05)$ as the level of COS in the diet was increased (Table 6). Also, in all the groups the antibody titers increased with the increasing of measurement time post vaccination. Similar trend was noticed when antibody titers to $P$. multocida were measured, showing higher titers $(\mathrm{P}<0.05)$ in animals fed COS diets.

At $54^{\text {th }}$ day, only MHC class II lymphocytes (quadratic; $\mathrm{P}=0.0725$ ) and $\mathrm{B}$-cells (quadratic; $\mathrm{P}=0.0815$ ) were higher in pigs offered COS supplemented diets (Table 7). The CD2, CD4, CD8, N cells and monocytes at $28^{\text {th }}$ or $54^{\text {th }}$ day were not influenced by the dietary treatments. 
Table 4. Effect of dietary supplementation of chito-oligosaccharides on the carcass characteristics and meat quality of the finishing pigs

\begin{tabular}{|c|c|c|c|c|c|c|c|}
\hline \multirow{2}{*}{\multicolumn{2}{|c|}{ Items }} & \multicolumn{3}{|c|}{ Treatments $^{1}$} & \multirow{2}{*}{$\mathrm{SEM}^{2}$} & \multicolumn{2}{|c|}{ P-value } \\
\hline & & control & T1 & $\mathrm{T} 2$ & & linear & quadratic \\
\hline \multicolumn{2}{|c|}{ Dressing percentage } & 74.10 & 71.18 & 72.93 & 0.84 & 0.5912 & 0.2376 \\
\hline \multicolumn{2}{|c|}{ Back fat thickness, mm } & 27.67 & 25.33 & 25.33 & 1.11 & 0.4582 & 0.6633 \\
\hline \multicolumn{2}{|c|}{ Lean, $\%$} & 52.56 & 53.99 & 55.34 & 0.74 & 0.1667 & 0.9825 \\
\hline \multicolumn{2}{|c|}{ Pork colour score } & 1.92 & 1.75 & 2.08 & 0.11 & 0.5442 & 0.3432 \\
\hline \multicolumn{2}{|c|}{ Marbling score } & 2.67 & 2.42 & 2.58 & 0.14 & 0.8382 & 0.5609 \\
\hline \multicolumn{8}{|c|}{ TBARS, $\mathrm{mg} / \mathrm{kg}$} \\
\hline & $0^{3}$ & 1.10 & 1.35 & 1.09 & 0.14 & 0.9794 & 0.4637 \\
\hline & 5 & 2.21 & 2.44 & 2.53 & 0.23 & 0.6317 & 0.9014 \\
\hline & 10 & 3.78 & 3.03 & 3.29 & 0.12 & 0.0048 & 0.0020 \\
\hline \multicolumn{8}{|c|}{ Drip loss, $\%$} \\
\hline & 5 & 4.15 & 5.76 & 4.67 & 0.40 & 0.5925 & 0.1429 \\
\hline & 10 & 16.44 & 10.75 & 7.41 & 1.87 & 0.0530 & 0.7315 \\
\hline \multicolumn{8}{|c|}{ CIE colour } \\
\hline \multirow[t]{3}{*}{$\mathrm{L}$} & 0 & 54.01 & 55.70 & 54.82 & 0.39 & 0.3950 & 0.1201 \\
\hline & 5 & 55.19 & 55.59 & 55.92 & 0.25 & 0.2479 & 0.9535 \\
\hline & 10 & 54.65 & 54.96 & 54.53 & 0.25 & 0.8427 & 0.5059 \\
\hline \multirow[t]{3}{*}{$\mathrm{a}$} & 0 & 16.29 & 16.69 & 17.63 & 0.29 & 0.0597 & 0.6542 \\
\hline & 5 & 15.51 & 15.07 & 15.23 & 0.16 & 0.4769 & 0.3879 \\
\hline & 10 & 15.46 & 15.93 & 17.12 & 0.35 & 0.0546 & 0.6246 \\
\hline \multirow[t]{3}{*}{$\mathrm{b}$} & 0 & 7.04 & 7.37 & 7.62 & 0.22 & 0.2783 & 0.9244 \\
\hline & 5 & 8.80 & 7.45 & 8.02 & 0.24 & 0.1871 & 0.0627 \\
\hline & 10 & 6.83 & 6.19 & 5.21 & 0.17 & 0.0001 & 0.5739 \\
\hline
\end{tabular}

${ }^{1}$ control: no chito-oligosaccharides; T1: chito-oligosaccharides added at $0.04 \%$ (30-55 kg), $0.02 \%$ $(55-85 \mathrm{~kg}), 0.02 \%(85-110 \mathrm{~kg})$, respectively; T2: chito-oligosaccharides added at $0.1 \%(30-55 \mathrm{~kg})$, $0.05 \%(55-85 \mathrm{~kg}), 0.025 \%(85-110 \mathrm{~kg})$, respectively.

${ }^{2}$ standard error of means

${ }^{3}$ day after post-mortem

Table 5. Effect of dietary supplementation of chito-oligosaccharides on pork cholesterol level in finishing pigs

\begin{tabular}{|c|c|c|c|c|c|c|}
\hline \multirow{2}{*}{ Items } & \multicolumn{3}{|c|}{ Treatments $^{1}$} & \multirow{2}{*}{$\mathrm{SEM}^{2}$} & \multicolumn{2}{|c|}{ P-value } \\
\hline & control & T1 & $\mathrm{T} 2$ & & linear & quadratic \\
\hline Total cholesterol, $\mathrm{mg} / 100 \mathrm{~g}$ & 53.92 & 50.74 & 49.61 & 2.10 & 0.4756 & 0.8414 \\
\hline $\mathrm{HDL}, \mathrm{mg} / 100 \mathrm{~g}$ & 18.04 & 21.35 & 23.73 & 2.38 & 0.4014 & 0.9353 \\
\hline $\mathrm{LDL}, \mathrm{mg} / 100 \mathrm{~g}$ & 35.88 & 29.40 & 25.89 & 2.14 & 0.0634 & 0.7089 \\
\hline
\end{tabular}

${ }^{1}$ control: no chito-oligosaccharides; T1: chito-oligosaccharides added at $0.04 \%$ (30-55 kg), $0.02 \%$ $(55-85 \mathrm{~kg}), 0.02 \%(85-110 \mathrm{~kg})$, respectively; T2: chito-oligosaccharides added at $0.1 \%(30-55 \mathrm{~kg})$, $0.05 \%(55-85 \mathrm{~kg}), 0.025 \%(85-110 \mathrm{~kg})$, respectively

${ }^{2}$ standard error of means 
Table 6. Effect of supplementation of chito-oligosaccharides on antibody titers to vaccine composed of A. pleuropneumoniae and P. multocida

\begin{tabular}{|c|c|c|c|c|c|c|}
\hline \multirow{2}{*}{ Items } & \multicolumn{3}{|c|}{ Treatments $^{1}$} & \multirow{2}{*}{$\mathrm{SEM}^{2}$} & \multicolumn{2}{|c|}{ P-value } \\
\hline & control & $\mathrm{T} 1$ & $\mathrm{~T} 2$ & & linear & quadratic \\
\hline \multicolumn{7}{|c|}{ A. pleuropneumoniae } \\
\hline D 0 & 6.60 & 8.00 & 18.66 & 2.14 & 0.0048 & 0.1016 \\
\hline D 5 & 16.00 & 38.00 & 51.20 & 6.82 & 0.0325 & 0.7032 \\
\hline D 30 & 170.60 & 198.00 & 234.60 & 10.88 & 0.0074 & 0.7533 \\
\hline D 50 & 298.60 & 469.30 & 490.60 & 34.82 & 0.0072 & 0.1232 \\
\hline D 70 & 938.60 & 1322.60 & 1536.00 & 104.64 & 0.0105 & 0.5673 \\
\hline \multicolumn{7}{|c|}{ P. multocida } \\
\hline D 0 & 6.60 & 9.30 & 14.70 & 1.44 & 0.0125 & 0.5234 \\
\hline D 5 & 15.30 & 31.30 & 42.00 & 4.63 & 0.0100 & 0.6857 \\
\hline D 30 & 21.30 & 38.80 & 54.70 & 5.83 & 0.0112 & 0.9237 \\
\hline D 50 & 29.30 & 45.30 & 85.30 & 9.76 & 0.0084 & 0.3732 \\
\hline D 70 & 74.70 & 86.00 & 101.30 & 4.66 & 0.0114 & 0.7655 \\
\hline
\end{tabular}

Table 7. Changes in the proportion of porcine leucocyte subpopulations at different intervals as affected by chito-oligosaccharides supplementation

\begin{tabular}{|c|c|c|c|c|c|c|}
\hline \multirow{2}{*}{ Items } & \multicolumn{3}{|c|}{ Treatments $^{1}$} & \multirow{2}{*}{$\mathrm{SEM}^{2}$} & \multicolumn{2}{|c|}{ P-value } \\
\hline & control & $\mathrm{T} 1$ & $\mathrm{~T} 2$ & & linear & quadratic \\
\hline \multicolumn{7}{|c|}{ MHC class II } \\
\hline D 0 & 18.48 & 17.44 & 16.06 & 1.03 & 0.4091 & 0.9450 \\
\hline D 28 & 23.30 & 16.82 & 14.80 & 1.97 & 0.1039 & 0.5829 \\
\hline D 54 & 25.07 & 32.08 & 29.50 & 1.36 & 0.1325 & 0.0725 \\
\hline \multicolumn{7}{|c|}{ CD2 + lymphocyte } \\
\hline D 0 & 41.66 & 44.25 & 50.38 & 2.35 & 0.1686 & 0.7263 \\
\hline D 28 & 32.33 & 34.13 & 40.93 & 1.98 & 0.8058 & 0.5177 \\
\hline D 54 & 39.01 & 34.38 & 39.30 & 2.00 & 0.9574 & 0.3285 \\
\hline \multicolumn{7}{|c|}{ CD4 + lymphocyte } \\
\hline D 0 & 20.64 & 22.66 & 24.16 & 1.25 & 0.3173 & 0.9291 \\
\hline D 28 & 9.53 & 10.83 & 14.94 & 1.27 & 0.1037 & 0.5883 \\
\hline D 54 & 7.47 & 7.13 & 9.02 & 0.57 & 0.3056 & 0.3889 \\
\hline \multicolumn{7}{|c|}{ CD8 + lymphocyte } \\
\hline D 0 & 22.27 & 29.79 & 23.70 & 2.00 & 0.7665 & 0.1384 \\
\hline D 28 & 19.95 & 17.10 & 24.73 & 1.57 & 0.1784 & 0.1019 \\
\hline D 54 & 28.14 & 23.07 & 24.56 & 1.50 & 0.3663 & 0.3418 \\
\hline
\end{tabular}


Table 7 continued

\begin{tabular}{|c|c|c|c|c|c|c|}
\hline \multirow{2}{*}{ Items } & \multicolumn{3}{|c|}{ Treatments $^{1}$} & \multirow{2}{*}{ SEM $^{2}$} & \multicolumn{2}{|c|}{ P-value } \\
\hline & control & $\mathrm{T} 1$ & $\mathrm{~T} 2$ & & linear & quadratic \\
\hline \multicolumn{7}{|c|}{ B lymphocyte } \\
\hline D 0 & 13.73 & 11.44 & 12.02 & 0.80 & 0.4328 & 0.4482 \\
\hline D 28 & 18.23 & 14.26 & 14.86 & 1.01 & 0.1878 & 0.2892 \\
\hline D 54 & 14.52 & 23.67 & 20.76 & 1.79 & 0.1104 & 0.0815 \\
\hline \multicolumn{7}{|l|}{$N$ cell } \\
\hline D 0 & 14.43 & 17.59 & 23.34 & 1.97 & 0.1032 & 0.7582 \\
\hline D 28 & 34.08 & 34.88 & 34.13 & 2.24 & 0.9939 & 0.8909 \\
\hline D 54 & 38.13 & 32.45 & 32.19 & 2.22 & 0.3318 & 0.5979 \\
\hline \multicolumn{7}{|l|}{ Monocyte } \\
\hline D 0 & 4.86 & 4.04 & 3.46 & 0.61 & 0.4181 & 0.9375 \\
\hline D 28 & 1.10 & 1.08 & 1.22 & 0.10 & 0.6766 & 0.7474 \\
\hline D 54 & 1.76 & 2.30 & 2.11 & 0.16 & 0.3839 & 0.3062 \\
\hline
\end{tabular}

${ }^{1}$ treatments: control, no chito-oligosaccharides; T1, chito-oligosaccharides added at $0.1 \%$; T2, chito-oligosaccharides added at $0.3 \%$

${ }^{2}$ standard error of means

\section{DISCUSSION}

Both chitin and chitosan are non-starch polysaccharides and have the potential to be regarded as components of dietary fibre. We were expecting a reduction in feed intake as found in weanling pigs in our previous study (Han et al., 2007), but it was not noticed in growing-finishing pigs, which could be partly explained that dietary fibre could be utilized more efficiently in growing-finishing pigs than the young pigs. The body weight of the pigs was not affected at any of the growing stages by COS addition. Kim et al. (1999) found that the growth performance was similar in growing and finishing pigs fed diet containing $0.03 \%$ of chitosan as compared with pigs fed diets with or without antibiotic. Pigs supplemented with $3 \mathrm{ml}$ of chitosan-alginate-Fe (II) complex in water attained market body weight 10 days earlier than those fed with control diet (Park et al., 2005). The addition of COS at the level of 0.2 and $0.1 \%$, respectively, in starter and grower diets of pigs, had no effect on the growth performance; however, the level of $0.3 \%$ reduced feed intake and improved feed:gain in weanling pigs (Han et al., 2007). These discrepancies in response to COS may result in part from the different molecular weights and different doses of the COS used in the experiments.

In the present study, the digestibility of $\mathrm{DM}, \mathrm{CP}, \mathrm{EE}, \mathrm{Ca}$ and $\mathrm{P}$ were not influenced by the addition of COS in the diets of pigs. However, linear and quadratic decrease in the digestibility of GE during early finisher and grower phases, respectively, due to addition of COS were noted. Higher nutrient digestibility in 
broiler chickens fed COS $(100 \mathrm{mg} / \mathrm{kg})$ supplemented diets have been reported (Huang et al., 2005; Li et al., 2007). Also, Han et al. (2007) had reported improvements in the DM and EE digestibility of weanling pigs fed COS $(0.4 \%$ from 6 to $13 \mathrm{~kg}$ and $0.3 \%$ from 13 to $30 \mathrm{~kg}$ ). However, Han et al. (2007) had conducted his experiment in weanling pigs and also the levels of COS used were higher than the levels of COS used in the present study which might partly explain the differences in the findings.

Meat or meat products are highly susceptible to lipid oxidation, which leads to rapid development of rancid or warmed-over flavour. Chitosan possesses antioxidant and antibacterial capacity, and may retard the lipid oxidation and inhibit the growth of spoilage bacteria in meat during storage (No et al., 2007). In the present study, although there was no difference in TBARS value of meat at day 0 and 5 of storage at $4^{\circ} \mathrm{C}$, both linear and quadratic reduction in TBARS values were noticed in COS added diets when compared with control. Darmadji and Izumimoto (1994a) had found that the beef added with $1.0 \%$ chitosan had $70 \%$ lower TBARS value after 3 days of storage at $4^{\circ} \mathrm{C}$ when compared with control. Moreover, at 10 days of storage the beef sample added with 0.5 and $1.0 \%$ chitosan had TBARS value same as that of 0 day, while there was a sharp increase in the TBARS value of control beef. Chitosan had a desirable effect on the development of the red colour of beef during storage. In the present study, the CIE colour of redness (a*) was significantly increased at d 10 of storage by COS supplementation, which was consistent with the findings by Darmadji and Izumimoto (1994b) who had reported improvement in the colour of minced beef added with $0.5 \%$ chitosan.

Chitosan acts as a weak anion exchange resin and exhibits a substantial viscosity in vitro. Either of these properties of chitosan could mediate its hypocholesterolaemic effect (Gallaher et al., 2000). In our study, lower LDL cholesterol concentrations were noticed in the pork of pigs fed with COS diets when compared with control however, there were no changes in the levels of total and HDL cholesterol. Cholesterol absorption was significantly reduced in layers and broilers fed chitosan compared with control group. Also, intragastric infusion of a lipid emulsion containing chitosan reduced lymphatic absorption of cholesterol. The mechanism for this effect might be the formation of gel complex by COS with gastric acid in the gastrointestinal tract and the gel complex cannot be degraded under the high $\mathrm{pH}$ environment in the intestine. This gel complex can adsorb bile acid and cholesterol and the gel, bile acid, cholesterol mixtures are discharged in faeces, thus the absorption of fat and cholesterol is decreased. Chitosans possess a positive ionic charge that gives it the ability to bond chemically with the negatively charged lipids, fats and bile acids. Chitosan reduced liver cholesterol in cholesterol-fed rats by decreasing cholesterol absorption and greatly increased the bile acid and fat excretion (Gallaher et al., 2000) and they further suggested that greater fat excretion should be explored as a means to decrease intestinal fat 
absorption. In the current study, fat digestibility was lowered in COS added diet during early finisher period possibly because of increased fat excretion by COS as suggested by previous studies and since there was less absorption, it may have reduced the cholesterol content in meat.

Many studies confirmed the effects of chitosan or COS on antitumor and improvement of immunity (Kim and Rajapakse, 2005) in vitro or in mice. However, this is the first report on immune studies with COS in pigs. Oligomers of chitin and chitosan are effective in enhancing migratory activity of macrophages and this activity can be explained by their chemotactic effects on macrophages. Furthermore, water-soluble chitosan can activate the production of cytokines such as IL- $1 \beta$, TNF- $\alpha$, and reactive oxygen intermediates to promote the defense system against microbial infections. In the present study, the antibody titers to $A$. pleuropneumoniae and P. multocida were higher in pigs fed COS diets when compared with the control and titers increased with the increase in measurement time post vaccination. The subset of porcine lymphocyte populations MHC class-II and B-cells showed higher lymphocytes only at $54^{\text {th }}$ day in COS supplemented diets as compared to pigs fed the control. Maeda and Kimura (2004) administered chitosan or oligochitosan in sarcoma 180-bearing mice and observed the reduction in the growth and final weight of tumor. In addition, the natural killer activity in intestinal intraepithelial and splenic lymphocytes was enhanced by chitosan or oligochitosan administration. Similarly, Wu et al. (2006) observed higher contents of IL-2, IL-4 and IL-6 and immune cells (white blood cells and lymphocytes) in chitosan administered mice as compared with control.

Overall, feeding chito-oligosaccharides in growing-finishing pigs improved pork stability and reduced cholesterol in pork without adverse effect on growth performance, and the immune response was improved by chito-oligosaccharides supplementation.

\section{REFERENCES}

AOAC, 1990. Association of Official Analytical Chemists, Official Methods of Analysis. $15^{\text {th }}$ Edition. Arlington, VA

Arvanitoyannis I.S., Nakayama A., Aiba S., 1998. Chitosan and gelatin based edible films: state diagrams, mechanical and permeation properties. Carbohyd. Polym. 37, 371-382

Darmadji P., Izumimoto M., 1994a. Effect of chitosan in meat preservation. Meat Sci. 38, 243-254

Darmadji P., Izumimoto M., 1994b. Effects of chitosan and nitrite on the properties of fermented meat. Anim. Sci. Technol. Jpn. 65, 639-646

Gallaher C.M., Munion J., Hesslink Jr. R., Wise J., Gallaher D.D., 2000. Cholesterol reduction by glucomannan and chitosan is mediated by changes in cholesterol absorption and bile acid and fat excretion in rats. J. Nutr. 130, 2753-2759

Han K.N., Kwon I.K., Lohakare J.D., Heo S., Chae B.J., 2007. Chito-oligosaccharides as an alternative to antimicrobials in improving performance, digestibility and microbial ecology of the gut in weanling pigs. Asian-Austr. J. Anim. Sci. 20, 556-562 
Huang R.L., Yin Y.L., Wu G.Y., Zhang Y.G., Li T.J., Li L.L., Li M.X., Tang Z.R., Zhang J., Wang B., He J.H., Nie X.Z., 2005. Effect of dietary oligochitosan supplementation on ileal digestibility of nutrients and performance in broilers. Poultry Sci. 84, 1383-1388

Ikeda I., Sugano M., Yoshida K., Sasaki E., Iwamoto Y., Hatano K., 1993. Effects of chitosan hydrolysates on lipid absorption and on serum and liver lipid concentration in rats. J. Agr. Food Chem. 41, 431-435

Kim J. D., Ko T. G., Han Y. K., Han I. K., 1999. Study on the development of antibiotic-free diet for growing-finishing pigs. Korean J. Anim. Nutr. Feed 23, 283-290

Kim S.K., Rajapakse N., 2005. Enzymatic production and biological activities of chitosan oligosaccharides (COS): A review. Carbohyd. Polym. 62, 357-368

Koo H.C., Ryu S.H., Ahn H.J., Jung W.K., Park Y.K., Kwon N.H., Kim S.H., Kim J.M., Yoo B.W., Choi S.I., Davis W.C., Park Y.H., 2006. Immunostimulatory effects of the anionic alkali mineral complex BARODON on equine lymphocytes. Clin. Vaccine Immunol. 13, 1255-1266

Li X.J., Piao X.S., Kim S.W., Liu P., Wang L., Shen Y.B., Jung S.C., Lee H.S., 2007. Effects of chitooligosaccharide supplementation on performance, nutrient digestibility, and serum composition in broiler chickens. Poultry Sci. 86, 1107-1114

Maeda Y., Kimura Y., 2004. Antitumor effects of various low-molecular-weight chitosans are due to increased natural killer activity of intestinal intraepithelial lymphocytes in sarcoma 180-bearing mice. J. Nutr. 134, 945-950

No H.K., Meyers S.P., Prinyawiwatkul W., Xu Z., 2007. Applications of chitosan for improvement of quality and shelf life of foods: a review. J. Food Sci. 72, R87-R100

NRC, 1998. Nutrient Requirements of Swine. 10 $0^{\text {th }}$ Edition. National Academy Press. Washington, DC

Park B.Y., Kim J.H., Cho S.H., Hwang I.H., Jung O.S., Kim Y.K., Lee J.M., Yun S.G., 2005. Effects of a dietary chitosan-alginate-fe(II) complex on meat quality of pig longissimus muscle during ageing. Asian-Austr. J. Anim. Sci. 18, 414-419

SAS, 1996. SAS User's Guide. Version 6.12. SAS Institute Inc. Cary, NC

Sinnhuber R.O., Yu T.C., 1977. The 2-thiobarbituric acid reaction, an objective measure of the oxidative determination occurring in fats and oils. J. Jpn. Soc. Fish Sci. 26, 259-267

Tang Z.R., Yin Y.L., Nyachoti C.M., Huang R.L., Li T.J., Yang C.B., Yang X.J., Gong J.S., Peng J., Qi D.S., Xiang J.J., Sun Z.H., Fan M.Z., 2005. Effect of dietary supplementation of chitosan and galacto-mannan-oligosaccharide on serum parameters and the insulin-like growth factor-I mRNA expression in early-weaned piglets. Domest. Anim. Endocrinol. 28, 430-441

Wu K.Y., Wu M., Fu M.L., Li H., Yang Y., Zhang H., Cheng C., Wang Z.Z., Wang X.Y., Lü X.B., Liu D.G., Li H., Gao R., 2006. A novel chitosan CpG nanoparticle regulates cellular and humoral immunity of mice. Biomed. Environ. Sci. 19, 87-95 